\title{
Perceived orientation in depth from line-of-sight movement
}

\author{
REX V. SLOCUM \\ Saint Francis College, Fort Wayne, Indiana 46808 \\ and \\ WAYNE A. HERSHBERGER \\ Northern Illinois University, De Kalb, Ilinois 60115
}

\begin{abstract}
Twelve college students viewed computer-generated displays of a cross comprised of two orthogonal dotted lines, and judged the apparent in-depth orientation of the horizontal arm by positioning a horizontal bar mounted on a rotary potentiometer. The vertical arm of the simulated cross was always in the observer's frontal plane, but the randomly textured horizontal arm was in one of nine orientations relative to the line of sight. Each observer viewed displays in which the simulated cross was, alternately, (a) stationary, (b) approaching the viewer, and (c) stationary but expanding in size. The static texture density gradient in the horizontal arm of the simulated stationary cross mediated perceived orientation in depth. Further, when motion perspective was added to the detail perspective, the impression of depth was enhanced, with the greatest enhancement obtaining at the near viewing distance. When dynamic magnification was added to the detail perspective, the impression of depth was attenuated; this effect was interpreted as an illusory case of motion perspective.
\end{abstract}

The present study sought to determine whether or not observers were sensitive to the information about an object's in-depth orientation that is contained in the motion perspective (Gibson, Olum, \& Rosenblatt, 1955) generated by the object's movement along the observer's line of sight.

A polar two-dimensional motion projection of an object translating a simulated line of sight contains motion perspective cues of movement in depth to which even very young observers are sensitive (e.g., Ball \& Tronick, 1971; Bower, Broughton, \& Moore, 1970). Further, Johansson (1964) has concluded from an experimental analysis of this phenomenon that transformation in both the $X$ and $Y$ dimensions of the simulated retinal projection appears to be a necessary condition for perceived translation in depth. But how motion perspective cues inherent in line-of-sight translation affect perceived in-depth orientation has not yet been determined. Braunstein (1966) has examined the perceived dimensionality of two- and three-dimensional stimuli as a function of their line-of-sight movement, but no one has previously examined the perceived in-depth orientation of an object as a function of such motion.

This report is based upon the doctoral dissertation of the first author and was supported by United States Public Health Service Research Grant 5 R01 EY00979-02 EXP from the National Eye Institute to Wayne A. Hershberger. A brief report of this research was presented at the Forty-Seventh Annual Meeting of the Midwestern Psychological Association, Chicago, 1975. Requests for reprints should be sent to Rex V. Slocum, Department of Psychology, Saint Francis College, Fort Wayne, Indiana 46808.
Braunstein (1966) presented his observers with motion pictures of computer-generated displays of either polar or parallel projections of randomly textured geometric forms (either two-dimensional or three-dimensional) which were in motion along (translation) or about (rotation) the $\mathrm{X}, \mathrm{Y}$, or $\mathrm{Z}$ axis (i.e., the horizontal axis, the vertical axis, and the line of sight, respectively). The observers were asked to indicate the direction of movement and the dimensionality (2-D or 3-D) of each display. Only the dimensionality judgments of the stimuli translating the $\mathrm{Z}$ axis need be considered here: The polar projections (simulating short viewing distances) yielded veridical dimensionality judgments of the three-dimensional stimulus but ambiguous dimensionality judgments of the frontally oriented two-dimensional stimulus. The parallel projections (simulating an infinite viewing distance) of $\mathrm{Z}$-axis translation incorporated no projected motion and, hence, appeared static, yielding ambiguous dimensionality judgments of the three-dimensional stimulus and veridical dimensionality judgments of the frontally oriented two-dimensional stimulus.

What is notable about these results is the ambiguous dimensionality of the polar projection of the frontally oriented two-dimensional figure. Since the "static" parallel projection of this figure was judged correctly, it appears that the motion perspective incorporated in the polar projection was in part an illusory depth cue. The present study, however, reveals that such motion perspective is also, in part, a veridical depth cue, one serving to mediate 
the perception of the in-depth orientation of such a two-dimensional object translating the line of sight.

In a static view of a uniformly textured surface, the visual angle subtended by an interstice separating adjacent elements of that surface varies as a function of both the viewing distance and the orientation of the surface. If, for example, a cross were formed in a frontal plane by two evenly dotted lines coincident with the $X$ and $Y$ axes, the sizes of the visual angles subtended by the (identical) interstices would vary with their distance from the origin (the intersection of $X$ and $Y$ ), the largest angles being subtended by the interstices closest to the origin. The angular texture density gradients in both the $\mathrm{X}$ and $\mathrm{Y}$ dimensions would be identical (i.e., simply rotated $90 \mathrm{deg}$ about the $\mathrm{Z}$ axis) and bilaterally symmetrical about the origin. If, however, the cross were rotated about the $Y$ axis, the angular texture density gradient in the $Y$ dimension would remain unchanged, and that of the $\mathrm{X}$ dimension would become bilaterally asymmetrical, the asymmetry increasing as a function of the deviation of the orientation of the horizontal arm from the frontal plane.

If the interstices of the horizontal arm were not uniform, but were random samples from some uniform population, the texture density gradient would be expressed as a gradient of probabilities of interstice sizes. The human retina is apparently sensitive to such gradients (e.g., Gibson, Gibson, Smith, \& Flock, 1959).

Translation of the cross along the line of sight would change the texture density gradients in the $X$ and $\mathrm{Y}$ dimensions, steepening them as the viewing distance decreased and flattening them as it increased. Thus, it might be expected that a dotted line oriented obliquely to the line of sight would more likely appear obliquely oriented at short viewing distances than at long ones.

Furthermore, it is possible that the dynamic transformations of the $X$-axis gradient may themselves mediate apparent in-depth orientation above and beyond that effected by any of the momentary static texture gradients incorporated in a polar motion projection, for Hershberger (1967) and others (e.g., Braunstein \& Payne, 1968; Jansson \& Borjesson, 1969) have demonstrated that the perceived direction of rotation in depth, which is related to apparent orientation in depth, is a function of the dynamic transformations within even a single dimension of the retinal projection.

Such a hypothesis might be evaluated by comparing orientation judgments made as the cross approaches the viewer (motion displays) with those made while the cross is stationary (static displays). In addition, the effect of changing angular size might be separated from the effect of changing angular texture gradient (both of which occur during Z-axis translation) by contrasting orientation judgments obtained from motion displays with those obtained from displays in which the texture gradient remained fixed while the display underwent size transformation comparable to the corresponding motion condition (magnification displays).

\section{METHOD}

\section{Observers}

Twelve experimentally naive college students (six male and six female) served as observers.

\section{Stimulus Displays}

Each observer viewed the face of a computer-controlled cathode ray tube (CRT). The computer was programmed to display polar projections of a simulated figure oriented in three-dimensional space. In each display, two dotted lines-one vertical and one horizontal-intersected at right angles in the center of the screen. Each display simulated a dotted cross situated in three-space with the vertical arm parallel to the face of the CRT and the other arm situated in a horizontal plane coincident with the center of the CRT screen.

The vertical and horizontal arms of the simulated cross each contained 21 dots (the center dot being common to both), although in certain displays not all of the dots were visible to the observer. Each of the 20 interstices of the vertical arm was $7.8 \mathrm{~mm}$, and the linear extent of the vertical arm was therefore $156 \mathrm{~mm}$. The interstices in the horizontal arm were of various sizes. They were selected at random from a uniform population ranging from .1 to $15.6 \mathrm{~mm}$, so that their expected value was $7.8 \mathrm{~mm}$, the size of the interstices in the vertical arm. The interstice lengths selected were $11.7 .8 .4,7.3 .5 .2,8.3,7.7,11.1,8.7,2.9,6.1,1.5,10.9,8.0,6.9$, $4.5,14.4,2.0,10.0,11.1$, and $4.9 \mathrm{~mm}$ (mean $=7.8 \mathrm{~mm}$; total length $151.6 \mathrm{~mm}$ ).

The simulated horizontal arm was situated in one of nine different orientations, or slants, relative to the optical, $\mathrm{Z}$, axis normal to the face of the CRT: $30,45,60,75,90,105,120,135$, and $150 \mathrm{deg}$. The nine orientations were combined factorially with 13 projection conditions grouped in three general classes: (a) motion, in which the simulated cross, in effect, moved toward the observer's vantage point, thus increasing both the absolute size of the display and steepening the texture gradient; (b) magnification (mag), in which the simulated cross, in effect, remained at a fixed projection distance while the absolute size of the display increased in size identical to the size increase of the motion displays; and (c) static, in which both the projection distance and absolute size of the cross remained fixed (i.e., the display was static).

There were two types of motion displays: (a) near, whose simulated viewing distance changed from 125 to $25 \mathrm{~mm}$ as the observers viewed it; and (b) far, whose simulated viewing distance changed from 225 to $125 \mathrm{~mm}$.

There were six types of magnification displays, three mimicking the size increase of motion/far and three mimicking the size increase of motion/near. Simultaneously, each magnification display exhibited a fixed interstice gradient correspondent to one of five projection distances. The six types of magnification displays were: (a) mag/1lear $/ 25 \mathrm{~mm}$. (b) mag/near $/ 75 \mathrm{~mm}$. (c) $\mathrm{mag} /$ near $/ 125 \mathrm{~mm}$. (d) $\mathrm{mag} / \mathrm{far} / 125 \mathrm{~mm}$, (e) $\mathrm{mag} / \mathrm{far} / 175 \mathrm{~mm}$, and (f) $\mathrm{mag} / \mathrm{far} / 225 \mathrm{~mm}$.

There were five types of static displays representing five fixed projection distances: (a) static $/ 25 \mathrm{~mm}$, (b) static $/ 75 \mathrm{~mm}$. (c) static $/ 125 \mathrm{~mm}$, (d) static $/ 175 \mathrm{~mm}$, and (e) static $/ 225 \mathrm{~mm}$.

\section{Apparatus}

The stimulus displays were presented on the face of a fast-phosphor CRT (VR-14) driven by a remote digital computer (PDP-12), both manufactured by Digital Equipment Corporation. The face of the CRT measured $17.8 \mathrm{~cm}$ high $\times 23.5 \mathrm{~cm}$ wide. Each 
dot in the stimulus cross measured approximately $.5 \mathrm{~mm}$ in diam and approximately $20 \mathrm{~mL}$ in luminance; the remainder of the screen was dark, i.e., did not luminesce. The dots were refreshed at a rate in excess of $1,000 / \mathrm{sec}$ so that they did not appear to flicker, and the resolution of the 512 by 512 addressable points on the screen was such that the apparent movement of the dots appeared continuous.

The displays were viewed monocularly, and indirectly through a monocular collimater providing a virtual image situated at infinity. The observer's eye was, hence, accommodated for infinity, and the lu minous textured cross was all that could be seen; neither the face of the CRT nor its borders were visible.

The collimator consisted of a rear-projection screen sandwiched between two lens systems which were mounted on a wooden support and situated at the seated observer's eye level. The lens system nearest the CRT screen projected the light rays from the CRT upon a $5.7-\mathrm{cm}$-square rear-projection screen. The rear-projection screen was situated in the first focal plane of a wide-angle $(68 \mathrm{deg})$ Erfle lens through which the observer viewed the image projected upon the rear-projection screen. This arrangement projected a viewing condition in which the visual angles of the displays were commensurate with the objective conditions simulated by the displays. For example, at the 90 -deg orientation, the horizontal arm of the simulated cross subtended visual angles of 38.4 and $64 \mathrm{deg}$, respectively. at projection distances of 225 and $125 \mathrm{~mm}$. At $25 \mathrm{~mm}$, the visual angle of the entire horizontal arm (normal to the $Z$ axis) would have been $144 \mathrm{deg}$, but the limiting effect of the projection apparatus restricted the observer's field of view to the center $68 \mathrm{deg}$. Thus, whenever the extremities of the cross exceeded a visual angle of $68 \mathrm{deg}$, they were not visible to the observer.

The observer indicated the apparent orientation of each display by positioning a horizontal aluminum bar $(1.3 \times 1.3 \times 15.2 \mathrm{~cm})$ mounted symmetrically onto the upper end of the vertical shaft of a potentiometer which, in turn. was mounted onto a tabletop $40.6 \mathrm{~cm}$ directly beneath the collimator. Immediately below the potentiometer was mounted a horizontal metal bar $1.3 \mathrm{~cm}$ wide, $.3 \mathrm{~cm}$ thick, and $5.7 \mathrm{~cm}$ long. The long axis of this bar was aligned parallel to the optical axis of the collimator and provided the observer with a kinesthetic spatial axis corresponding to the optical axis. The observer held the reference bar in one hand and the potentiometer bar in the other. He did not view either bar; a curtain suspended from the wooden frame supporting the collimator shielded the observer's hands from his view. The potentiometer, wired to the computer, was linear, with the value of one on the computer's voltmeter corresponding to approximately $.4 \mathrm{deg}$ of rotation.

Since the stimulus displays were presented in the visual mode and judgments were expressed in the haptic mode, the observers were obliged, as intended, to judge the relevant stimulus feature common to both, namely, orientation in depth. Further, although the raw data reflect the joint effects of visual and haptic judgments, differences among the data for different viewing conditions (i.e., static, motion, and magnification) reflect the uncontaminated effects of those viewing conditions, since the mode of responding remained constant across viewing conditions.

\section{Procedure}

The observers viewed the displays individually in two $1-\mathrm{h}$ sessions scheduled on consecutive days. Each observer viewed the simulated cross in all nine orientations under each of the 13 projection conditions during each of the two experimental sessions. In a single session, each motion display was viewed twice, and each magnification and static display was viewed once. Thus, each observer made a total of 135 observations (and responses) per session: 36 motion, 54 magnification, and 45 static. The order of presentation of the $\mathbf{1 3 5}$ displays was determined randomly for each observer, and the displays were viewed in reverse sequence during the second session, 1 day later.

The observers were instructed to look into the eyepiece, where they would see two dotted lines forming a cross, the horizontal arm of which, over a series of trials, would be in a variety of orientations.
They were told that their task was to set the aluminum bar (in their hands) to the same orientation as that of the horizontal arm of the cross, saying "OK" to the experimenter each time they accomplished the task, so that he could record the response and initiate the next presentation. On the second day, the observer was told that the task was identical to that of the first day, but that the order of presentation of displays had been changed.

\section{RESULTS}

The statistical analyses described below were not performed upon the raw data, which were judgments of the orientation of the stimulus figure's horizontal arm on each trial, but upon derived data, which were averages of the response judgments across several trials. In the motion displays, the stimulus figure had been presented at continuously changing projection distances, but in the magnification and static displays the projection distances were fixed. In order to obtain judgments of the magnification and static displays from a comparable range of projection distances, it was necessary to calculate mean judgments across several trials (i.e., three trials), where on each trial the stimulus figure had been projected from a particular distance, and the various distances, as a group, constituted a representative sample of the projection distances incorporated in the corresponding motion display. That is, the three distances corresponded to the 0,50 , and 100 percentiles of the range of distances incorporated in the corresponding motion display. Specifically, these distances were 25,75 , and $125 \mathrm{~mm}$ for the near projection distances and 125, 175, and $225 \mathrm{~mm}$ for the far projection distances.

Each magnification and static display was presented once during the experimental session, but the motion displays were presented twice. The responses from these two presentations were averaged, yielding a single derived judgment for each of the motion/near and motion/far displays.

Table 1

Overall Analysis of Variance: Summary of Significant Effects Among Constant Error Scores

\begin{tabular}{lrrrr}
\hline \multicolumn{1}{c}{ Source } & \multicolumn{1}{c}{ MS } & df & $\begin{array}{l}\text { Error } \\
\text { Term }\end{array}$ & \multicolumn{1}{c}{ F } \\
\hline Angle & $142,748.1$ & 8 & OA(X) & $638.54^{*}$ \\
Sex by Angle & $1,737.4$ & 8 & OA(X) & $7.77^{*}$ \\
OA(X) & 223.6 & 80 & & \\
Projection by Angle & $1,780.9$ & 16 & OPA(X) & $21.88^{*}$ \\
$\begin{array}{l}\text { OPA(X) } \\
\text { Distance by Angle }\end{array}$ & 81.4 & 160 & & \\
$\begin{array}{l}\text { ODA(X) } \\
\text { Projection by Distance }\end{array}$ & 475.0 & 8 & ODA(X) & $13.75^{*}$ \\
$\quad$ by Angle & 329.8 & 80 & & \\
OPDA(X) & 48.6 & 160 & & \\
\hline
\end{tabular}

Note-The abbreviations designate the following factors: $A=$ angle, $D=$ projection distance, $O=$ observer, $P=$ type of projection, and $X=$ sex. $O P A(X)$, for example, indicates observer by projection by angle, within sex.

${ }^{*} p<.001$ 


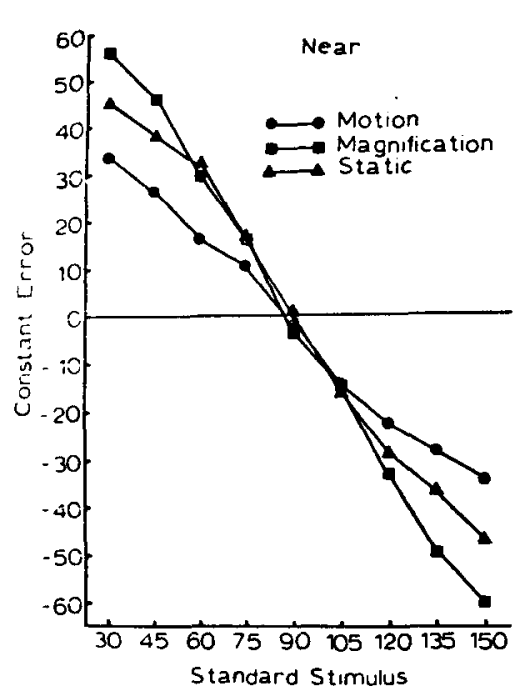

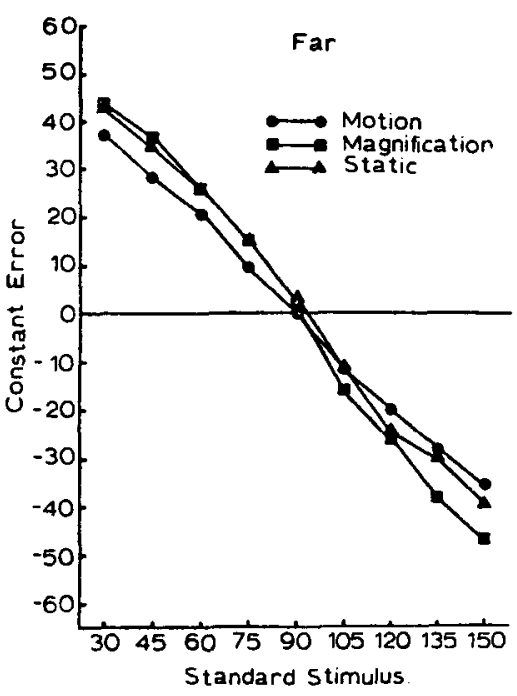

Figure 1. Constant error for each standard stimulus under each viewing condition at each projection distance (Projection by Distance by Angle interaction).
The derived judgments were arranged in a five-way factorial design with six replications (observers) per cell, the five factors being sex, experimental session (first, second), projection (motion, magnification, static), distance (near, far), and angle $(30,45,60,75$, $90,105,120,135$, and $150 \mathrm{deg}$ ).

The constant error of each of the derived judgments was determined by subtracting from each judgment the value of the corresponding standard stimulus.
These constant error scores were then subjected to an overall analysis of variance which yielded five significant $(\mathrm{p}<.01)$ effects: angle, Projection by Angle, Distance by Angle, Sex by Angle, and Projection by Distance by Angle. A summary of the significant effects is given in Table 1 . The three-way interaction, Projection by Distance by Angle, is presented in Figures 1 and 2. Figure 1 illustrates the interaction directly, by showing the mean constant
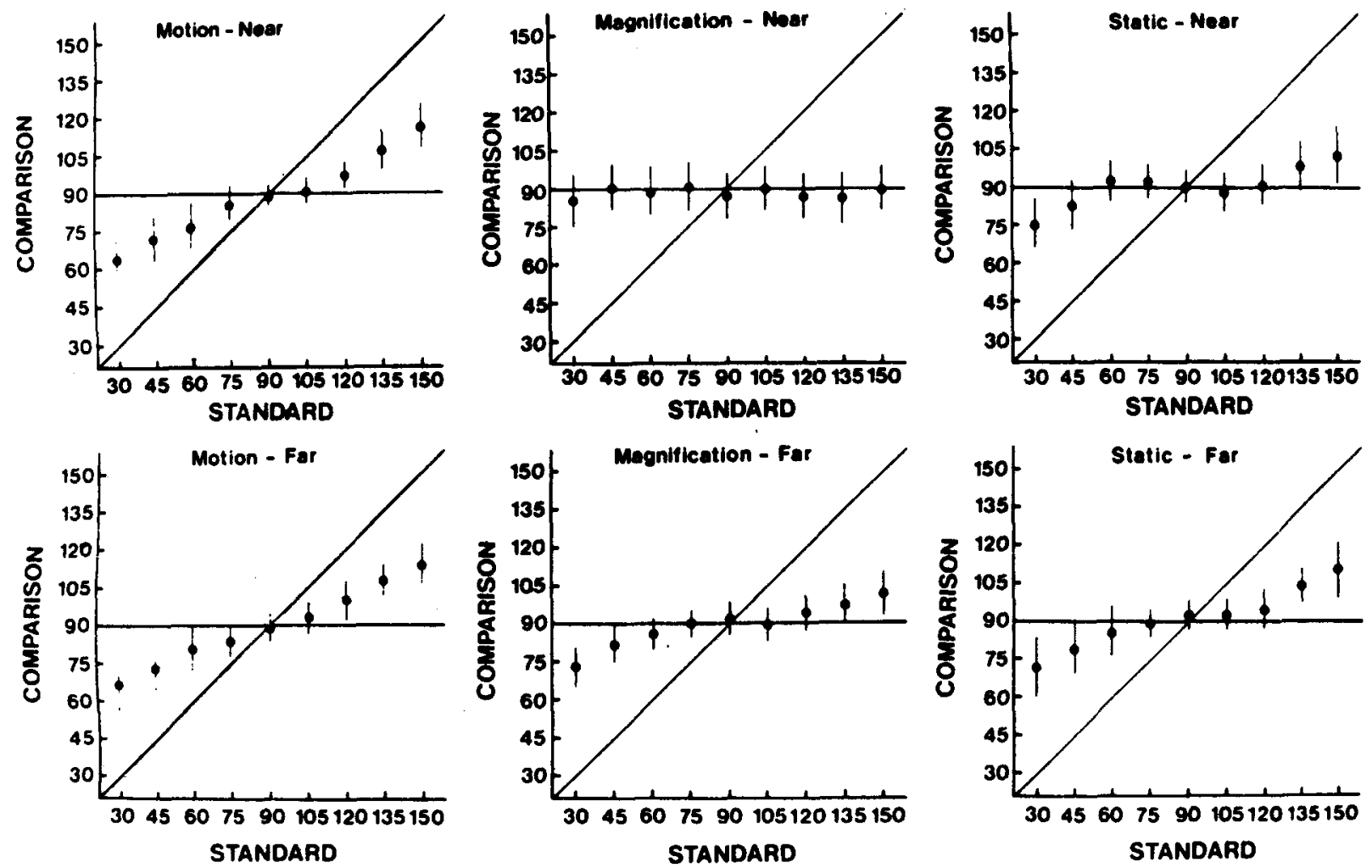

Figure 2. Mean judgments for each viewing condition at each projection distance. The vertical line through each mean represents the variable error associated with that mean, and the vertical distance from the mean to the diagonal represents the constant error associated with that mean. 
Table 2

Summary of Simple Interactions Comprising Projection by Distance by Angle Interaction of Constant Error Scores

\begin{tabular}{lrrc}
\hline Source & MS & df & \multicolumn{1}{c}{$F$} \\
\hline Distance by Angle & & & \\
$\quad$ Motion & 45.3 & 8 & 1.08 \\
Magnification & 903.0 & 8 & $21.60^{*}$ \\
Static & 286.2 & 8 & $6.85^{*}$ \\
ODA(X) & 41.8 & 80 & \\
Projection by Angle & & & \\
Near & $1,756.1$ & 16 & $21.58^{*}$ \\
Far & 354.7 & 16 & $4.36^{*}$ \\
Near: Motion, magnification & $3,339.0$ & 8 & $41.03^{*}$ \\
$\quad$ Motion, static & $1,162.3$ & 8 & $14.28^{*}$ \\
$\quad$ Magnification, static & 767.0 & 8 & $9.42^{*}$ \\
Far: Motion, magnification & 684.0 & 8 & $8.41^{*}$ \\
$\quad$ Motion, static & 223.4 & 8 & 2.75 \\
$\quad$ Magnification, static & 156.5 & 8 & 1.92 \\
OPA(X) & 81.4 & 160 & \\
\hline
\end{tabular}

Note-OPA $(X)$ and $O D A(X)$ indicate, respectively, observer by projection by angle, within sex, and observer by distance by angle, within sex. These error terms were derived in the overall analysis of variance.

${ }^{*} p<.001$

error (averaged across observers, sex, and session), while Figure 2 shows the mean derived judgments (points of subjective equality) averaged across observers, sex, and session.

A summary of the analyses of the simple interactions comprising the Projection by Distance by Angle interaction is presented in Table 2. The Distance by Angle interactions were significant $(p<.001)$ for the magnification and for the static displays, but not for the motion displays. That is to say, the increasing constant error associated with increasing angles of inclination of the standard stimulus from the frontal plane differed at the near and far projection distances for the magnification and static displays, but not for the motion displays. In the magnification and static displays, the mean constant error increased faster with increasing obliqueness of the standard stimulus at the near than at the far projection distance, while in the motion displays the functions relating mean constant error to stimulus figure obliqueness at the near and far projection distances were the same.

The simple Projection by Angle interactions were significant at each level of distance (near, far), and they were further simplified by assessing the significance of the Projection by Angle interactions using all possible combinations of two projection conditions (motion and magnification, motion and static. and magnification and static) at each level of distance. Of these six simple Projection by Angle interactions, which are presented in Table 2, each of the three at the near distance was significant, but at the far distance only the two interactions containing motion judgments were significant. Thus, at the near projection distance, the increase in mean constant error with increasing obliqueness of the standard stimulus differed significantly for all three projection conditions: the general slope of the increasing function was greatest in the magnification displays and least in the motion displays, with the static displays yielding a slope intermediate to the other two. At the far projection distance, the rate of change in constant error across levels of angle was the same for the magnification and static conditions, and it was greater than the rate of change associated with the motion displays.

Figure 2 presents the mean derived judgments (i.e., the points of subjective equality) for each angle under each type of projection at each projection distance. The diagonal on each graph represents the scores of the ideal observer (i.e., errorless performance) and the vertical distance between the point of subjective equality (PSE) and the diagonal represents constant error. The between-observers variable error associated with each PSE (derived by averaging the absolute deviations of each of the responses from the corresponding PSE) is indicated by the vertical line extending above and below each data point. An analysis of variance on the variable error scores indicated a significant Projection by Angle interaction, $F(16,160)=2.07, p<.025$, which is presented in Figure 3, but not a significant main

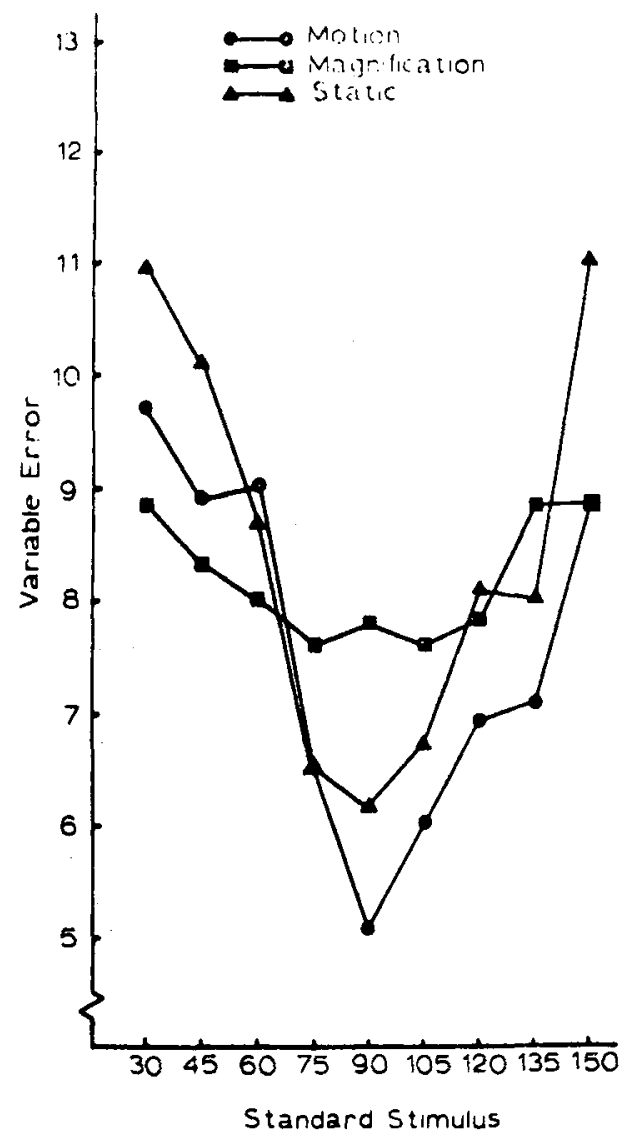

Figure 3. Mean variable error for each projection at each angle (Projection by Angle interaction). 
effect for projection, $F(2,20)=2.49, \mathrm{p}>.10$. Subsequent analysis of the three possible combinations of two levels of projection indicated that the functions relating variable error to stimulus figure orientation were not significantly different for the motion and static displays, $F(8,160)=.80, p>.25$; but they were signiticantly different for the magnification and motion displays, $F(8,160)=2.36$, $\mathrm{p}<.025$, and for the magnification and static displays, $F(8,160)=3.04, p<.005$. Thus, the between-observers variable error increased equally in the motion and static conditions with increasing obliqueness of the standard stimulus, and the slope of this function was greater than that of the magnification condition. At the frontal and near-frontal orientations of the stimulus figure, the variable error was greater for the magnification displays than for the motion and static displays, while the reverse was generally true at the extreme angles of inclination of the stimulus figure from the frontal plane.

\section{DISCUSSION}

Dynamic change in the visual angles subtended by the texture elements of an object translating depth affects the perceived in-depth orientation of the object. For, in the present study, the perceptual effects which are not attributable to static density perspective must be attributed to the dynamic cues (i.e., motion perspective) incorporated in the displays.

The mean judgments of the static displays varied as a positive function of the orientation of the stimulus figure, indicating that the texture density gradient mediated orientation in depth. Since the slope of the function of the static-far displays was steeper than that of the static-near displays, the texture density gradient was apparently more salient at the far distance than at the near. This is not to say that static detail perspective is generally more salient at greater viewing distances. Very likely the inferior performance with the static-near displays resulted from the fact that only a portion of the nearest static lines (subtending up to as much as $144 \mathrm{deg}$ ) was visible to the observer looking through the 68-deg collimator.

The constant error of the motion-display judgments increased with increasing obliqueness of the stimulus figure, and the motion-near and motion-far functions relating constant error to stimulus orientation did not differ. The constant error of the motion displays, however, increased less than that of the static displays with increasing obliqueness of the standard stimulus. This decrease in constant error was not accompanied by a differential increase in variable error, since the change in variable error, across angles, was the same for the motion and static displays. In other words, the perceived orientation of the motion displays, relative to that of the static displays, was more veridical at steep angles, and this relative superiority in mean response accuracy was not accompanied by (i.e., was not at the expense of) increased variability of the judgments.

In polar projections of $\mathrm{Z}$-axis translation, the projected texture elements of the stimulus figure's horizontal arm move away from the $X, Y$ origin at angular velocities which vary as a function of their distance from the origin. (For a mathematical analysis of such projections, see Gibson, Olum, and Rosenblatt, 1955.) If a texture element exists at the origin, which Gibson (1950) has called the focus of expansion, it remains stationary, but the angular velocities of the successive texture elements form a gradient (i.e., a transformation gradient) across the projection. When the stimulus figure is oriented in the frontal plane, the transformation gradient in the projection is bilaterally symmetrical about the origin, with the angular velocities first increasing and then decreasing with increased distance from the origin. But, when the figure is oblique to the $Z$ axis, the transformation gradient is asymmetrical about the origin and the texture elements in the portion of the figure nearer the projection point move at greater angular velocities than the corresponding elements in the portion of the figure farther from the projection point. The degree of bilateral asymmetry in the transformation gradient is a function of the orientation of the stimulus figure and of the projection distance.

When the projection distances are large, relative to the lateral extent of the stimulus figure's horizontal arm, the bilateral asymmetry in the transformation gradient is small. At shorter projection distances, the bilateral asymmetry is correspondingly larger; and therefore the saliency of motion perspective as a cue to orientation in depth would be expected to be an inverse function of the projection distance. It is this increased saliency of the motion perspective at the near distance, relative to the far, which apparently offset the decreased saliency of the detail perspective and resulted in the motion-near judgments being as veridical as the motion-far judgments.

It might be argued that the superior performance elicited by the motion displays was not due to motion perspective cues, but to the fact that an optimal projection distance which yields a "best" percept of orientation in depth was always momentarily exhibited in the motion displays, and that the three projection distances used to derive the judgments of the static-near and static-far displays, respectively, did not each manifest this optimal projection distance, resulting in a less-veridical average performance. In an effort to assess this possibility, the performance elicited by the static displays at the projection distance resulting in the smallest constant error (i.e., static $/ 175 \mathrm{~mm}$ ) was contrasted with the 
performance of the motion displays at the distance yielding the largest constant error (i.e., motion/near). The mean absolute constant error of the motion/near condition was significantly less than that of the static $/ 175-\mathrm{mm}$ condition, $\mathrm{t}(120)=2.02, \mathrm{p}<.025$, $S E_{\text {diff }}=1.40$, demonstrating that the superior performance of the motion displays is not due to their inclusion of a "best" projection, but to motion perspective cues provided by the dynamic transformation of the projection. That is, motion perspective is a veridical cue to the in-depth orientation of the stimulus figure translating the $\mathrm{Z}$ axis. To say that it is a veridical cue is not to say that the percept it mediates is veridical. The judgments reflect considerable constant error involving regression to the frontal plane.

In contrast to the transformation gradient of the motion displays, the angular velocities of the texture elements in the magnification displays, at both the near and the far projection distances, were uniform across the projection. Regardless of the orientation of the stimulus figure, the transformation gradient of the magnification displays was always bilaterally symmetrical and, hence, equivalent to the motion perspective of a frontal-plane cross moving in depth. Thus, while the texture density gradient in the magnification displays was a function of the orientation of the stimulus figure (i.e., it was a veridical cue), the angular velocities of the texture elements were independent of the orientation of the stimulus figure and always represented the motion perspective of a cross aligned in a frontal plane.

Since the motion perspective cues in the motion displays were found to be more salient at the near than at the far projection distances, the illusory effect of the pseudomotion perspective in the magnification displays would be expected to be greater at the near than at the far projection distance. And since the saliency of the detail perspective cues varied as an increasing function of the projection distance, it might be expected that the magnification/near displays, incorporating salient illusory cues (motion perspective) and weak veridical cues (detail perspective) would elicit less accurate judgments of the stimulus figure's orientation than the static/near displays, which incorporated only the weak veridical cues (detail perspective). At the far projection distance, however, this difference between the static and magnification judgments should be smaller, since the illusory motion perspective in magnification displays would be attenuated and the veridical detail perspective would be equally salient in both the magnification and static displays. Such was the case: At both the near and far projection distances, the constant error of the magnification judgments was a function of the obliqueness of the standard stimulus, and the slope of this function was greater at the near projection distance than at the far. The mean judgments elicited by the magnification/near displays did not vary with stimulus figure orientation as the static/near judgments did, indicating that the perceived orientation of the magnification/near displays was a function of the (apparently illusory) motion perspective cues rather than of the veridical detail perspective cues. At the far projection distance, however, the mean judgments of both the static and magnification displays were functions of the stimulus figure orientation, and these functions did not differ from one another. This means that the difference in veridicality between the near and far projection distances was greater for the magnification than for the static projections, confirming the prediction.

Although Z-axis translation of a two-dimensional figure may tend to yield an illusory appearance of three-dimensionality, as Braunstein (1966) has shown, it tends also to yield a veridical impression of the in-depth orientation of that figure, even when the motion perspective is confined to a single dimension of the retinal projection.

\section{REFERENCES}

Ball, W., \& Tronick, E. Infant responses to impending collision: Optical and real. Science. 1971, 171, 818-820.

Bower, T. G. R.. Broughton, J. M., \& Moore, M. K. Infant responses to approaching objects: An indicator of response to distal variables. Perception \& Psychophysics, 1970, 9, 193-196.

Braunstein, M. L. Sensitivity of the observer to transformations of the visual field. Journal of Experimental Psychology, 1966, 72, 683-687.

Braunstein, M. L., \& Payne, J. W. Perspective and the rotating trapezoid. Joumal of the Optical Society of America. 1968. 58. 399-403.

Grbson, E. J., Gibson, J. J., Smith, O. W., \& Flock, H. Motion parallax as a determinant of perceived depth. Journal of Experimental Psychology, 1959, 58, 40-51.

Gibson. J. J. The perception of the visual world. Boston: Houghton Mifflin, 1950.

Gibson, J. J., Olum, P., \& Rosenblatt, F. Parallax and perspective during aircraft landings. American Journal of Psychology, 1955, 68, 372-385.

Hershberger, W. A. Comment on "Apparent reversal (oscillation) of rotary motion in depth." Psychological Review, 1967. 74, 235-238.

Jansson, G., \& Bonjesson, E. Perceived direction of rotary motion. Perception \& Psychophysics, 1969, 6, 19-26.

Johansson, G. Perception of motion and changing form. Scandinavian Journal of Psychology, 1964, 5, 181-208.

(Received for publication May 20, 1975; revision accepted October 20, 1975.) 